\title{
ARTICLE OPEN \\ Identification and tunable optical coherent control of transition-metal spins in silicon carbide
}

Tom Bosma ${ }^{1}$, Gerrit J. J. Lof ${ }^{1}$, Carmem M. Gilardoni ${ }^{1}$, Olger V. Zwier ${ }^{1}$, Freddie Hendriks ${ }^{1}$, Björn Magnusson ${ }^{2,3}$, Alexandre Ellison ${ }^{3}$, Andreas Gällström ${ }^{2,4}$, Ivan G. Ivanov ${ }^{2}$, N. T. Son ${ }^{2}$, Remco W. A. Havenith ${ }^{1,5,6}$ and Caspar H. van der Wal (iD)

Color centers in wide-bandgap semiconductors are attractive systems for quantum technologies since they can combine longcoherent electronic spin and bright optical properties. Several suitable centers have been identified, most famously the nitrogenvacancy defect in diamond. However, integration in communication technology is hindered by the fact that their optical transitions lie outside telecom wavelength bands. Several transition-metal impurities in silicon carbide do emit at and near telecom wavelengths, but knowledge about their spin and optical properties is incomplete. We present all-optical identification and coherent control of molybdenum-impurity spins in silicon carbide with transitions at near-infrared wavelengths. Our results identify spin $S=1 / 2$ for both the electronic ground and excited state, with highly anisotropic spin properties that we apply for implementing optical control of ground-state spin coherence. Our results show optical lifetimes of $\sim 60 \mathrm{~ns}$ and inhomogeneous spin dephasing times of $\sim 0.3 \mu \mathrm{s}$, establishing relevance for quantum spin-photon interfacing.

npj Quantum Information (2018)4:48 ; doi:10.1038/s41534-018-0097-8

\section{INTRODUCTION}

Electronic spins of lattice defects in wide-bandgap semiconductors have come forward as an important platform for quantum technologies, ${ }^{1}$ in particular for applications that require both manipulation of long-coherent spin and spin-photon interfacing via bright optical transitions. In recent years this field showed strong development, with demonstrations of distribution and storage of non-local entanglement in networks for quantum communication ${ }^{2-6}$ and quantum-enhanced field-sensing. ${ }^{7-11}$ The nitrogen-vacancy defect in diamond is the material system that is most widely used ${ }^{12,13}$ and best characterized ${ }^{14-16}$ for these applications. However, its zero-phonon-line (ZPL) transition wavelength $(637 \mathrm{~nm})$ is not optimal for integration in standard telecom technology, which uses near-infrared wavelength bands where losses in optical fibers are minimal. A workaround could be to convert photon energies between the emitter-resonance and telecom values, ${ }^{17-19}$ but optimizing these processes is very challenging.

This situation has been driving a search for similar lattice defects that do combine favorable spin properties with bright emission directly at telecom wavelength. It was shown that both diamond and silicon carbide ( $\mathrm{SiC}$ ) can host many other spin-active color centers that could have suitable properties ${ }^{20-23}$ (where $\mathrm{SiC}$ is also an attractive material for its established position in the semiconductor device industry ${ }^{24,25}$ ). However, for many of these color centers detailed knowledge about the spin and optical properties is lacking. In SiC the divacancy ${ }^{26-28}$ and silicon vacancy ${ }^{10,29-31}$ were recently explored, and these indeed show millisecond homogeneous spin coherence times with bright ZPL transitions closer to the telecom band.

We present here a study of transition-metal impurity defects in $\mathrm{SiC}$, which exist in great variety. ${ }^{32-37}$ There is at least one case (the vanadium impurity) that has ZPL transitions at telecom wavelengths, ${ }^{33}$ around $1300 \mathrm{~nm}$, but we focus here (directed by availability of lasers in our lab) on the molybdenum impurity with ZPL transitions at $1076 \mathrm{~nm}$ (in $4 \mathrm{H}-\mathrm{SiC}$ ) and $1121 \mathrm{~nm}$ (in $6 \mathrm{H}-\mathrm{SiC}$ ), which turns out to be a highly analogous system. Theoretical investigations, ${ }^{38}$ early electron paramagnetic resonance ${ }^{33,39}$ (EPR), and photoluminescence (PL) studies ${ }^{40-42}$ indicate that these transition-metal impurities have promising properties. These studies show that they are deep-level defects that can be in several stable charge states, each with a distinctive value for its electronic spin $S$ and near-infrared optical transitions. Further tuning and engineering possibilities come from the fact that these impurities can be embedded in a variety of SiC polytypes $(4 \mathrm{H}, 6 \mathrm{H}$, etc., Fig. 1a). Recent work by Koehl et al. ${ }^{37}$ studied chromium impurities in $4 \mathrm{H}-\mathrm{SiC}$ using optically detected magnetic resonance. They identified efficient ZPL (little phonon-sideband) emission at $1042 \mathrm{~nm}$ and $1070 \mathrm{~nm}$, and their charge state as neutral with an electronic spin $S=1$ for the ground state.

Our work is an all-optical study of ensembles of molybdenum impurities in p-type $4 \mathrm{H}-\mathrm{SiC}$ and $6 \mathrm{H}-\mathrm{SiC}$ material. The charge and spin configuration of these impurities, and the defect configuration in the $\mathrm{SiC}$ lattice that is energetically favored, was until our work not yet identified with certainty. Our results show that these Mo impurities are in the $\mathrm{Mo}^{5+}\left(4 \mathrm{~d}^{1}\right)$ charge state (we follow here conventional notation: ${ }^{33}$ the label $5+$ indicates that of an original

\footnotetext{
${ }^{1}$ Zernike Institute for Advanced Materials, University of Groningen, NL-9747AG Groningen, The Netherlands; ${ }^{2}$ Department of Physics, Chemistry and Biology, Linköping University, SE-581 83 Linköping, Sweden; ${ }^{3}$ Norstel AB, Ramshällsvägen 15, SE-602 38 Norrköping, Sweden; ${ }^{4}$ Saab Dynamics AB, SE-581 88 Linköping, Sweden; ${ }^{5}$ Stratingh Institute for

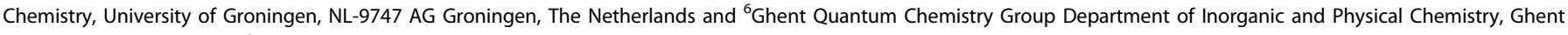
University, B-9000 Gent, Belgium
}

Correspondence: Tom Bosma (tom.bosma@rug.nl) or Caspar H. van der Wal (c.h.van.der.wal@rug.nl)

These authors contributed equally: Tom Bosma, Gerrit J. J. Lof.

Received: 24 April 2018 Revised: 27 August 2018 Accepted: 30 August 2018

Published online: 01 October 2018 
a

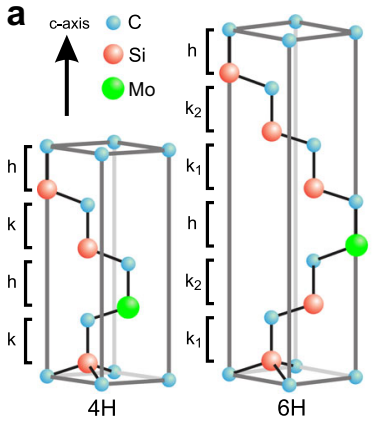

b

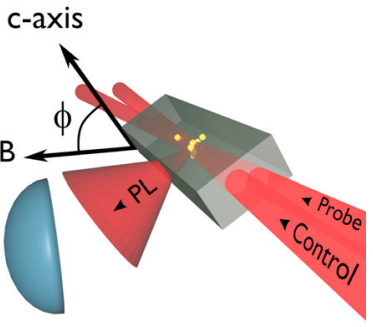

PL (PLE) collection

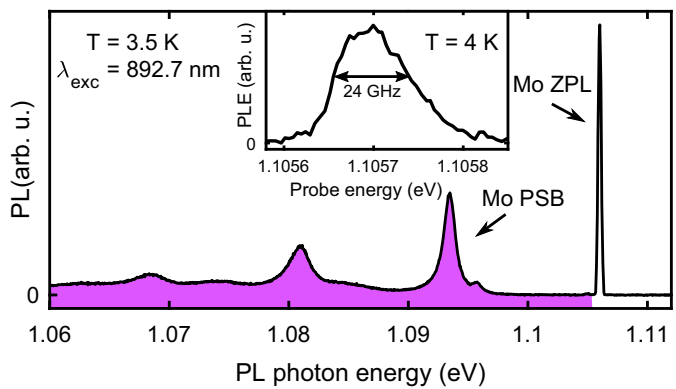

Fig. 1 Crystal structures of $\mathrm{SiC}$, setup schematic and optical signatures of $\mathrm{Mo}$ in $6 \mathrm{H}-\mathrm{SiC}$ a Schematic illustration of the stacking of Si-C bilayers in the crystal structure of the $4 \mathrm{H}-\mathrm{SiC}$ and $6 \mathrm{H}-\mathrm{SiC}$ polytypes, which gives lattice sites with cubic and hexagonal local environment labeled by $k_{(1,2)}$ and $h$, respectively. Our work revisits the question whether Mo impurities are present as substitutional atoms (as depicted) or residing inside $\mathrm{Si}-\mathrm{C}$ divacancies. The $c$-axis coincides with the growth direction. b Schematic of SiC crystal in the setup. The crystal is placed in a cryostat with optical access. Laser excitation beams (control and probe for two-laser experiments) are incident on a side facet of the SiC crystal and propagate normal to the $c$-axis. Magnetic fields $\mathbf{B}$ are applied in a direction orthogonal to the optical axis and at angle $\phi$ with the c-axis. Photoluminescence (PL) is collected and detected out of another side facet of the $\mathrm{SiC}$ crystal. c PL from Mo in $6 \mathrm{H}-\mathrm{SiC}$ at $3.5 \mathrm{~K}$ and zero field, resulting from excitation with an $892.7 \mathrm{~nm}$ laser, with labels identifying the zero-phonon-line ( $Z \mathrm{PL}$, at $1.1057 \mathrm{eV})$ emission and phonon replicas (shaded and labeled as phonon sideband, PSB). The inset shows the ZPL as measured by photoluminescence excitation (PLE). Here, the excitation laser is scanned across the ZPL peak and emission from the PSB is used for detection

Mo atom 4 electrons participate in bonds with $\mathrm{SiC}$ and that 1 electron is transferred to the p-type lattice environment). The single remaining electron in the $4 d$ shell gives spin $S=1 / 2$ for the ground state and optically excited state that we address. While we will show later that this can be concluded from our measurements, we assume it as a fact from the beginning since this simplifies the explanation of our experimental approach.

In addition to this identification of the impurity properties, we explore whether ground-state spin coherence is compatible with optical control. Using a two-laser magneto-spectroscopy method, ${ }^{28,43,44}$ we identify the spin Hamiltonian of the $S=1 / 2$ ground state and optically excited state, which behave as doublets with highly anisotropic Landé g-factors. This gives insight in how a situation with only spin-conserving transitions can be broken, and we find that we can use a weak magnetic field to enable optical transitions from both ground-state spin levels to a common excited-state level ( $\Lambda$ level scheme). Upon two-laser driving of such $\Lambda$ schemes, we observe coherent population trapping (CPT, all-optical control of ground-state spin coherence and fundamental to operating quantum memories ${ }^{45,46}$ ). The observed CPT reflects inhomogeneous spin dephasing times comparable to that of the SiC divacancy ${ }^{28,47}$ (near $1 \mu \mathrm{s}$ ).
In what follows, we first present our methods and results of single-laser spectroscopy performed on ensembles of Mo impurities in both SiC polytypes. Next, we discuss a two-laser method where optical spin pumping is detected. This allows for characterizing the spin sublevels in the ground and excited state, and we demonstrate how this can be extended to controlling spin coherence.

Both the $6 \mathrm{H}-\mathrm{SiC}$ and $4 \mathrm{H}-\mathrm{SiC}$ (Fig. 1a) samples were intentionally doped with Mo. There was no further intentional doping, but nearband-gap photoluminescence revealed that both materials had $\mathrm{p}$ type characteristics. The Mo concentrations in the $4 \mathrm{H}$ and $6 \mathrm{H}$ samples were estimated ${ }^{41,42}$ to be in the range $10^{15}-10^{17} \mathrm{~cm}^{-3}$ and $10^{14}-10^{16} \mathrm{~cm}^{-3}$, respectively. The samples were cooled in a liquid-helium flow cryostat with optical access, which was equipped with a superconducting magnet system. The set-up geometry is depicted in Fig. 1b. The angle $\phi$ between the direction of the magnetic field and the $c$-axis of the crystal could be varied, while both of these directions were kept orthogonal to the propagation direction of excitation laser beams. In all experiments where we resonantly addressed ZPL transitions the laser fields had linear polarization, and we always kept the direction of the linear polarization parallel to the $c$-axis. Earlier studies $s^{38,41,42}$ of these materials showed that the ZPL transition dipoles are parallel to the $c$-axis. For our experiments we confirmed that the photoluminescence response was clearly the strongest for excitation with linear polarization parallel to the $c$-axis, for all directions and magnitudes of the magnetic fields that we applied. All results presented in this work come from photoluminescence $(\mathrm{PL})$ or photoluminescence excitation (PLE) measurements. The excitation lasers were focused to a $\sim 100 \mu \mathrm{m}$ spot in the sample. PL emission was measured from the side. A more complete description of experimental aspects is presented in Methods section.

\section{RESULTS}

For initial characterization of Mo transitions in $6 \mathrm{H}-\mathrm{SiC}$ and $4 \mathrm{H}-\mathrm{SiC}$ we used PL and PLE spectroscopy (Methods). Figure 1c shows the $\mathrm{PL}$ emission spectrum of the $6 \mathrm{H}-\mathrm{SiC}$ sample at $3.5 \mathrm{~K}$, measured using an $892.7 \mathrm{~nm}$ laser for excitation. The ZPL transition of the Mo defect visible in this spectrum will be studied in detail throughout this work. The shaded region indicates the emission of phonon replicas related to this ZPL. ${ }^{41,42}$ While we could not perform a detailed analysis, the peak area of the ZPL in comparison with that of the phonon replicas indicates that the ZPL carries clearly more than a few percent of the full PL emission. Similar PL data from Mo in the $4 \mathrm{H}-\mathrm{SiC}$ sample, together with a study of the temperature dependence of the $\mathrm{PL}$, can be found in Supplementary Information (Fig. S1).

For a more detailed study of the ZPL of the Mo defects, PLE was used. In PLE measurements, the photon energy of a narrowlinewidth excitation laser is scanned across the ZPL part of the spectrum, while resulting PL of phonon-sideband (phonon-replica) emission is detected (Fig. 1b, we used filters to keep light from the excitation laser from reaching the detector, Methods). The inset of Fig. 1c shows the resulting ZPL for $\mathrm{Mo}$ in $6 \mathrm{H}-\mathrm{SiC}$ at $1.1057 \mathrm{eV}$ $(1121.3 \mathrm{~nm})$. For $4 \mathrm{H}-\mathrm{SiC}$ we measured the $\mathrm{ZPL}$ at $1.1521 \mathrm{eV}$ (1076.2 nm, Supplementary Information). Both are in close agreement with literature. ${ }^{41,42}$ Temperature dependence of the PLE from the Mo defects in both $4 \mathrm{H}-\mathrm{SiC}$ and $6 \mathrm{H}-\mathrm{SiC}$ can be found in Supplementary Information (Fig. S2).

The width of the ZPL is governed by the inhomogeneous broadening of the electronic transition throughout the ensemble of Mo impurities, which is typically caused by non-uniform strain in the crystal. For Mo in $6 \mathrm{H}-\mathrm{SiC}$ we observe a broadening of $24 \pm$ $1 \mathrm{GHz}$ FWHM, and $23 \pm 1 \mathrm{GHz}$ for $4 \mathrm{H}-\mathrm{SiC}$. This inhomogeneous broadening is larger than the anticipated electronic spin splittings, $^{33}$ and it thus masks signatures of spin levels in optical transitions between the ground and excited state. 
a

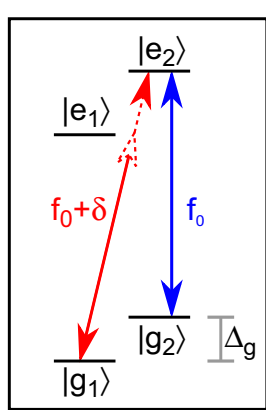

b

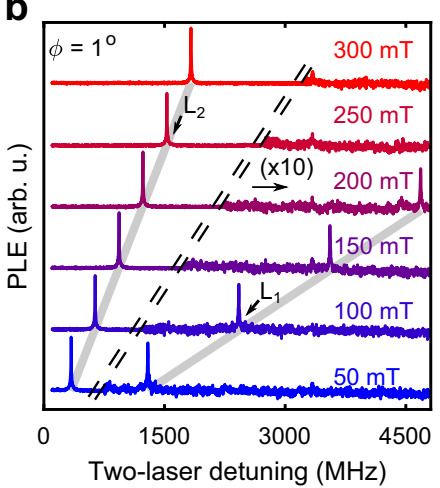

C

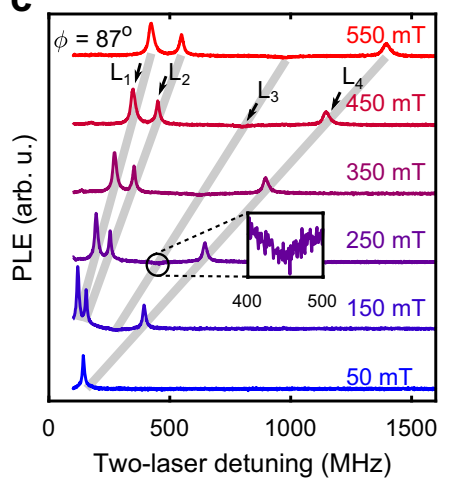

d

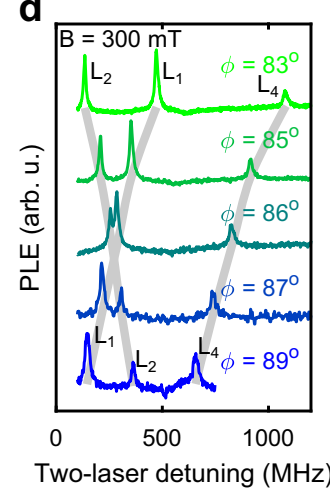

Fig. 2 Two-laser spectroscopy results for Mo in $6 \mathrm{H}-\mathrm{SiC}$. a Working principle of two-laser spectroscopy: one laser at frequency $f_{0}$ is resonant with the $\left|g_{2}\right\rangle-\left|e_{2}\right\rangle$ transition, the second laser is detuned from the first laser by $\delta$. If $\delta$ is such that the second laser becomes resonant with another transition (here sketched for $\left|g_{1}\right\rangle-\left|e_{2}\right\rangle$ ) the photoluminescence will increase since optical spin-pumping by the first laser is counteracted by the second and vice versa. b-d Photoluminescence excitation (PLE) signals as a function of twolaser detuning at $4 \mathrm{~K}$. b Magnetic field dependence with field parallel to the $c$-axis $\left(\phi=1^{\circ}\right)$. For clarity, data in the plot have been magnified by a factor 10 right from the dashed line. Two peaks are visible, labeled $L_{1}$ and $L_{2}$ (the small peak at $3300 \mathrm{MHz}$ is an artefact from the Fabry-Pérot interferometer in the setup). c Magnetic field dependence with the field nearly perpendicular to the $c$-axis ( $\phi=$ $87^{\circ}$ ). Three peaks and a dip (enlarged in the inset) are visible. These four features are labeled $L_{1}$ through $L_{4}$. The peak positions as a function of field in $\mathbf{b}, \mathbf{c}$ coincide with straight lines through the origin (within $0.2 \%$ error). $\mathbf{d}$ Angle dependence of the PLE signal at $300 \mathrm{mT}$ (angles accurate within $2^{\circ}$ ). Peaks $L_{1}$ and $L_{4}$ move to the left with increasing angle, whereas $L_{2}$ moves to the right. The data in bd are offset vertically for clarity

In order to characterize the spin-related fine structure of the Mo defects, a two-laser spectroscopy technique was employed. ${ }^{28,43,44}$ We introduce this for the four-level system sketched in Fig. 2a. A laser fixed at frequency $f_{0}$ is resonant with one possible transition from ground to excited state (for the example in Fig. 2a $\left|g_{2}\right\rangle$ to $\mid$ $\left.\left.e_{2}\right\rangle\right)$, and causes PL from a sequence of excitation and emission events. However, if the system decays from the state $\left|e_{2}\right\rangle$ to $\left|g_{1}\right\rangle$, the laser field at frequency $f_{0}$ is no longer resonantly driving optical excitations (the system goes dark due to optical pumping). In this situation, the PL is limited by the (typically long) lifetime of the $\left|g_{1}\right\rangle$ state. Addressing the system with a second laser field, in frequency detuned from the first by an amount $\delta$, counteracts optical pumping into off-resonant energy levels if the detuning $\delta$ equals the splitting $\Delta_{g}$ between the ground-state sublevels. Thus, for specific two-laser detuning values corresponding to the energy spacings between ground-state and excited-state sublevels the PL

response of the ensemble is greatly increased. Notably, this technique gives a clear signal for sublevel splittings that are smaller than the inhomogeneous broadening of the optical transition, and the spectral features now reflect the homogeneous linewidth of optical transitions. ${ }^{28,47}$

In our measurements a $200 \mu \mathrm{W}$ continuous-wave control and probe laser were made to overlap in the sample. For investigating $\mathrm{Mo}$ in $6 \mathrm{H}-\mathrm{SiC}$ the control beam was tuned to the $\mathrm{ZPL}$ at $1121.32 \mathrm{~nm}\left(f_{\text {control }}=f_{0}=267.3567 \mathrm{THz}\right)$, the probe beam was detuned from $f_{0}$ by a variable detuning $\delta$ (i.e., $f_{\text {probe }}=f_{0}+\delta$ ). In addition, a $100 \mu \mathrm{W}$ pulsed $770 \mathrm{~nm}$ re-pump laser was focused onto the defects to counteract bleaching of the Mo impurities due to charge-state switching ${ }^{28,48,49}$ (which we observed to only occur partially without re-pump laser). All three lasers were parallel to within $3^{\circ}$ inside the sample. A magnetic field was applied to ensure that the spin sublevels were at non-degenerate energies. Finally, we observed that the spectral signatures due to spin disappear in a broad background signal above a temperature of $\sim 10 \mathrm{~K}$ (Fig. S4), and we thus performed measurements at $4 \mathrm{~K}$ (unless stated otherwise).

Figure $2 \mathrm{~b}$ shows the dependence of the PLE on the two-laser detuning for the $6 \mathrm{H}-\mathrm{SiC}$ sample $(4 \mathrm{H}-\mathrm{SiC}$ data in Supplementary Information Fig. S6), for a range of magnitudes of the magnetic field (here aligned close to parallel with the $c$-axis, $\phi=1^{\circ}$ ). Two emission peaks can be distinguished, labeled line $L_{1}$ and $L_{2}$. The emission (peak height) of $L_{2}$ is much stronger than that of $L_{1}$. Figure 2c shows the results of a similar measurement with the magnetic field nearly orthogonal to the crystal c-axis $\left(\phi=87^{\circ}\right)$, where four spin-related emission signatures are visible, labeled as lines $L_{1}$ through $L_{4}$ (a very small peak feature left from $L_{1}$, at half its detuning, is an artifact that occurs due to a leakage effect in the spectral filtering that is used for beam preparation, see Methods). The two-laser detuning frequencies corresponding to all four lines emerge from the origin $(\mathbf{B}=0, \delta=0)$ and evolve linearly with magnetic field (we checked this up to 1.2 T). The slopes of all four lines (in Hertz per Tesla) are smaller in Fig. 2c than in Fig. 2b. In contrast to lines $L_{1}, L_{2}$, and $L_{4}$, which are peaks in the PLE spectrum, $L_{3}$ shows a dip.

In order to identify the lines at various angles $\phi$ between the magnetic field and the c-axis, we follow how each line evolves with increasing angle. Figure $2 \mathrm{~d}$ shows that as $\phi$ increases, $L_{1}, L_{3}$, and $L_{4}$ move to the left, whereas $L_{2}$ moves to the right. Near $86^{\circ}, L_{2}$ and $L_{1}$ cross. At this angle, the left-to-right order of the emission lines is swapped, justifying the assignment of $L_{1}, L_{2}, L_{3}$, and $L_{4}$ as in Fig. 2b, c. Supplementary Information presents further results from two-laser magneto-spectroscopy at intermediate angles $\phi$ (section 2a).

We show below that the results in Fig. 2 indicate that the Mo impurities have electronic spin $S=1 / 2$ for the ground and excited state. This contradicts predictions and interpretations of initial results. $33,38,41,42$ Theoretically, it was predicted that the defect associated with the ZPL under study here is a Mo impurity in the asymmetric split-vacancy configuration (Mo impurity asymmetrically located inside a $\mathrm{Si}-\mathrm{C}$ divacancy), where it would have a spin $S=1$ ground state with zero-field splittings of about $3-6 \mathrm{GHz}$. $^{33,38,41,42}$ However, this would lead to the observation of additional emission lines in our measurements. Particularly, in the presence of a zero-field splitting, we would expect to observe twolaser spectroscopy lines emerging from a non-zero detuning. ${ }^{28} \mathrm{We}$ have measured near zero fields and up to $1.2 \mathrm{~T}$, as well as from $100 \mathrm{MHz}$ to $21 \mathrm{GHz}$ detuning (Supplementary Information section $2 \mathrm{c}$ ), but found no more peaks than the four present in Fig. 2c. A larger splitting would have been visible as a splitting of the ZPL in measurements as presented in the inset of Fig. 1c, which was not observed in scans up to $1000 \mathrm{GHz}$. Additionally, a zero-field splitting and corresponding avoided crossings at certain magnetic fields would result in curved behavior for the positions of lines in magneto-spectroscopy. Thus, our observations rule out that there 
a

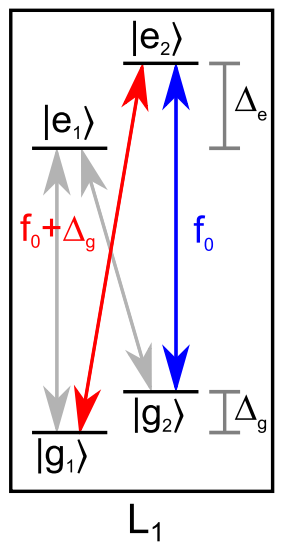

b

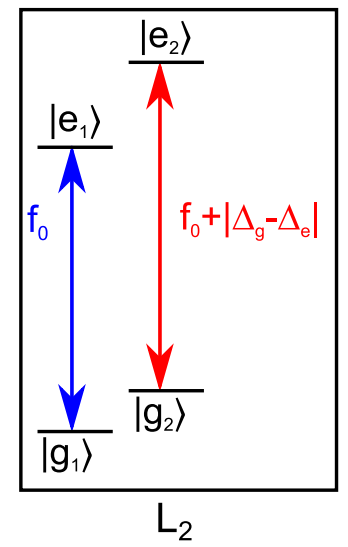

C

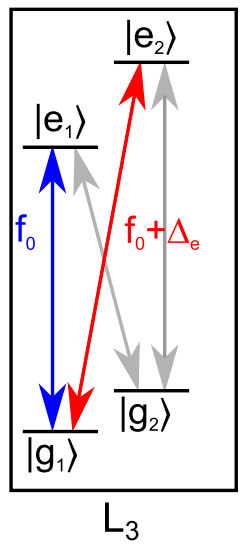

d

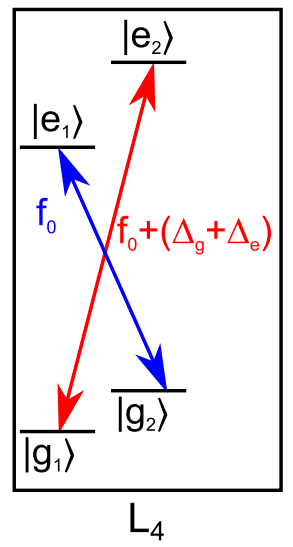

Fig. 3 Two-laser pumping schemes with optical transitions between $S=1 / 2$ ground and excited states. a $\Lambda$ scheme, responsible for $L_{1}$ emission feature: Two lasers are resonant with transitions from both ground states $\left|g_{1}\right\rangle$ (red arrow) and $\left|g_{2}\right\rangle$ (blue arrow) to a common excited state $\left|e_{2}\right\rangle$. This is achieved when the detuning equals the ground-state splitting $\Delta_{g}$. The gray arrows indicate a secondary $\Lambda$ scheme via $\left|e_{1}\right\rangle$ that is simultaneously driven in an ensemble when it has inhomogeneous values for its optical transition energies. $\mathbf{b} \Pi$ scheme, responsible for $L_{2}$ emission feature: Two lasers are resonant with both vertical transitions. This is achieved when the detuning equals the difference between the ground-state and excited-state splittings, $\left|\Delta_{g}-\Delta_{e}\right| . \mathrm{c} V$ scheme, responsible for $L_{3}$ emission feature: Two lasers are resonant with transitions from a common ground state $\left|g_{1}\right\rangle$ to both excited states $\left|e_{1}\right\rangle$ (blue arrow) and $\left|e_{2}\right\rangle$ (red arrow). This is achieved when the laser detuning equals the excited state splitting $\Delta_{e}$. The gray arrows indicate a secondary $\mathrm{V}$ scheme that is simultaneously driven when the optical transition energies are inhomogeneously broadened. $\mathbf{d} X$ scheme, responsible for the $L_{4}$ emission feature: Two lasers are resonant with the diagonal transitions in the scheme. This is achieved when the detuning is equal to the sum of the ground-state and the excited-state splittings, $\left(\Delta_{g}+\Delta_{e}\right)$

is a zero-field splitting for the ground-state and excited-state spin sublevels. In this case the effective spin-Hamiltonian ${ }^{50}$ can only take the form of a Zeeman term

$H_{g(e)}=\mu_{B} g_{g(e)} \mathbf{B} \cdot \tilde{\mathbf{S}}$,

where $g_{g(e)}$ is the g-factor for the electronic ground (excited) state (both assumed positive), $\mu_{B}$ the Bohr magneton, B the magnetic field vector of an externally applied field, and $\tilde{\mathbf{S}}$ the effective spin vector. The observation of four emission lines can be explained, in the simplest manner, by a system with spin $S=1 / 2$ (doublet) in both the ground and excited state.

For such a system, Fig. 3 presents the two-laser optical pumping schemes that correspond to the observed emission lines $L_{1}$ through $L_{4}$. Addressing the system with the V-scheme excitation pathways from Fig. $3 c$ leads to increased pumping into a dark ground-state sublevel, since two excited states contribute to decay into the off-resonant ground-state energy level while optical excitation out of the other ground-state level is enhanced. This results in reduced emission observed as the PLE dip feature of $L_{3}$ in Fig. 2c (for details see Supplementary Information section 5).

We find that for data as in Fig. $2 c$ the slopes of the emission lines are correlated by a set of sum rules

$\Theta_{L 3}=\Theta_{L 1}+\Theta_{L 2}$

$\Theta_{L 4}=2 \Theta_{L 1}+\Theta_{L 2}$

Here $\Theta_{L n}$ denotes the slope of emission line $L_{n}$ in Hertz per Tesla. The two-laser detuning frequencies for the pumping schemes in Fig. 3a-d are related in the same way, which justifies the assignment of these four schemes to the emission lines $L_{1}$ through $L_{4}$, respectively. These schemes and equations directly yield the $g$-factor values $g_{g}$ and $g_{e}$ for the ground and excited state (Supplementary Information section 2).

We find that the g-factor values $g_{g}$ and $g_{e}$ strongly depend on $\phi$, that is, they are highly anisotropic. While this is in accordance with earlier observations for transition metal defects in $\mathrm{SiC}^{33}$ we did not find a comprehensive report on the underlying physical picture. In Supplementary Information section 7, we present a group-theoretical analysis that explains the anisotropy $g_{g} \approx 1.7 \mathrm{for}$

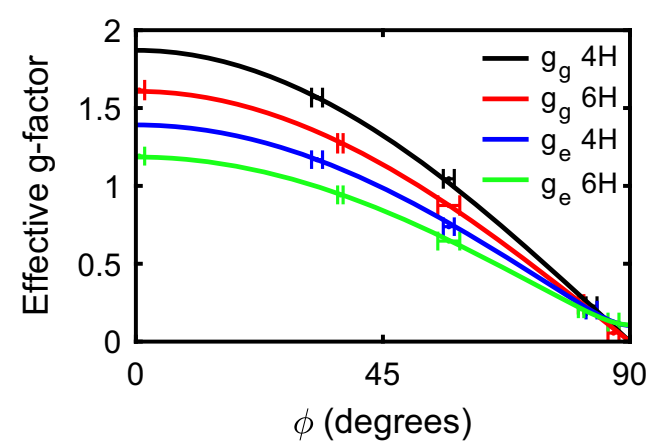

Fig. 4 Effective g-factors for the spin of Mo impurities in SiC. Angular dependence of the $g$-factor for the $S=1 / 2$ ground $\left(g_{g}\right)$ and excited states $\left(g_{e}\right)$ of the Mo impurity in $4 \mathrm{H}-\mathrm{SiC}$ and $6 \mathrm{H}-\mathrm{SiC}$. The solid lines indicate fits of Eq. (4) to the data points extracted from twolaser magneto-spectroscopy measurements as in Fig. 2b,c

$\phi=0^{\circ}$ and $g_{g}=0$ for $\phi=90^{\circ}$, and similar behavior for $g_{e}$ (which we also use to identify the orbital character of the ground and excited state). In this scenario the effective Landé $\mathrm{g}$-factor ${ }^{50}$ is given by

$g(\phi)=\sqrt{\left(g_{\|} \cos \phi\right)^{2}+\left(g_{\perp} \sin \phi\right)^{2}}$,

where $g_{\|}$represents the component of $g$ along the $c$-axis of the silicon carbide structure and $g_{\perp}$ the component in the basal plane. Figure 4 shows the ground and excited state effective g-factors extracted from our two-laser magneto-spectroscopy experiments for $6 \mathrm{H}-\mathrm{SiC}$ and $4 \mathrm{H}-\mathrm{SiC}$ (additional experimental data can be found in Supplementary Information). The solid lines represent fits to the Eq. (4) for the effective g-factor. The resulting $g_{\|}$and $g_{\perp}$ parameters are given in Table 1.

The reason why diagonal transitions (in Fig. 3a, c), and thus the $\Lambda$ and $V$ scheme are allowed, lies in the different behavior of $g_{e}$ and $g_{g}$. When the magnetic field direction coincides with the internal quantization axis of the defect, the spin states in both the ground and excited state are given by the basis of the $S_{z}$ operator, 


\begin{tabular}{|lcl|}
\hline $\begin{array}{l}\text { Table 1. Components of the } \mathrm{g} \text {-factors for the spin of Mo impurities in } \\
\mathrm{SiC}\end{array}$ & $g_{\perp}$ \\
\hline & & \\
\hline $4 \mathrm{H}-\mathrm{SiC}$ & $1.87 \pm 0.2$ & $0.04 \pm 0.04$ \\
Ground state & $1.39 \pm 0.2$ & $0.10 \pm 0.02$ \\
Excited state & & \\
GH-SiC & $1.61 \pm 0.02$ & $0.000 \pm 0.004$ \\
Ground state & $1.20 \pm 0.02$ & $0.11 \pm 0.02$ \\
Excited state &
\end{tabular}

where the $z$-axis is defined along the $c$-axis. This means that the spin-state overlap for vertical transitions, e.g., from $\left|g_{1}\right\rangle$ to $\left|e_{1}\right\rangle$, is unity. In such cases, diagonal transitions are forbidden as the overlap between e.g., $\left|g_{1}\right\rangle$ and $\left|e_{2}\right\rangle$ is zero. Tilting the magnetic field away from the internal quantization axis introduces mixing of the spin states. The amount of mixing depends on the $\mathrm{g}$-factor, such that it differs for the ground and excited state. This results in a tunable non-zero overlap for all transitions, allowing all four schemes to be observed (as in Fig. 2b where $\phi=87^{\circ}$ ). This reasoning also explains the suppression of all emission lines except $L_{2}$ in Fig. $2 \mathrm{~b}$, where the magnetic field is nearly along the $c$ axis. A detailed analysis of the relative peak heights in Fig. $2 b, c$ compared to wave function overlap can be found in Supplementary Information (section 4).

The $\Lambda$ driving scheme depicted in Fig. 3a, where both ground states are coupled to a common excited state, is of particular interest. In such cases it is possible to achieve all-optical coherent population trapping (CPT) ${ }^{45}$ which is of great significance in quantum-optical operations that use ground-state spin coherence. This phenomenon occurs when two lasers address a $\Lambda$ system at exact two-photon resonance, i.e., when the two-laser detuning matches the ground-state splitting. The ground-state spin system is then driven toward a superposition state that approaches $\left|\Psi_{\text {CPT }}\right\rangle \propto \Omega_{2}\left|g_{1}\right\rangle-\Omega_{1}\left|g_{2}\right\rangle$ for ideal spin coherence. Here $\left|\Omega_{n}\right\rangle$ is the Rabi frequency for the driven transition from the $\left|g_{n}\right\rangle$ state to the common excited state. Since the system is now coherently trapped in the ground state, the photoluminescence decreases.

In order to study the occurrence of CPT, we focus on the twolaser PLE features that result from a $\Lambda$ scheme. A probe field with variable two-laser detuning relative to a fixed control laser was scanned across this line in frequency steps of $50 \mathrm{kHz}$, at $200 \mu \mathrm{W}$. The control laser power was varied between $200 \mu \mathrm{W}$ and $5 \mathrm{~mW}$. This indeed yields signatures of CPT, as presented in Fig. 5. A clear power dependence is visible: when the control beam power is increased, the depth of the CPT dip increases (and can fully develop at higher laser powers or by concentrating laser fields in $\mathrm{SiC}$ waveguides ${ }^{47}$ ). This observation of CPT confirms our earlier interpretation of lines $L_{1}-L_{4}$, in that it confirms that $L_{1}$ results from a $\wedge$ scheme. It also strengthens the conclusion that this system is $S=1 / 2$, since otherwise optical spin-pumping into the additional (dark) energy levels of the ground state would be detrimental for the observation of CPT.

Using a standard model for $\mathrm{CPT}^{45}$ adapted to account for strong inhomogeneous broadening of the optical transitions ${ }^{47}$ (see also Supplementary Information section 6) we extract an inhomogeneous spin dephasing time $T_{2}^{*}$ of $0.32 \pm 0.08 \mu \mathrm{s}$ and an optical lifetime of the excited state of $56 \pm 8 \mathrm{~ns}$. The optical lifetime is about a factor two longer than that of the nitrogenvacancy defect in diamond, ${ }^{12,51}$ indicating that the Mo defects can be applied as bright emitters (although we were not able to measure their quantum efficiency). The value of $T_{2}^{*}$ is relatively short but sufficient for applications based on CPT. ${ }^{45}$ Moreover, the EPR studies by Baur et al. ${ }^{33}$ on various transition-metal impurities show that the inhomogeneity probably has a strong static

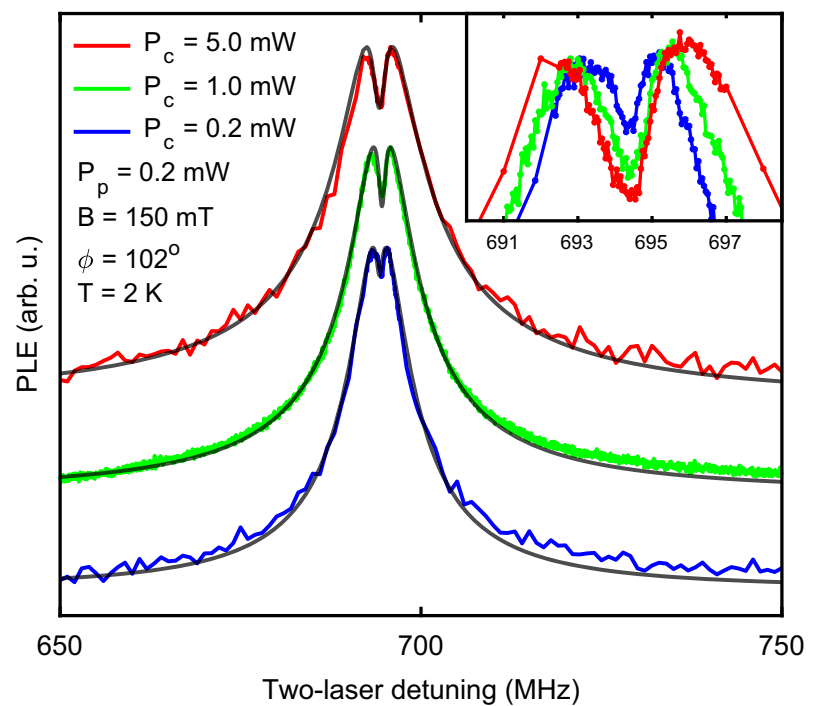

Fig. 5 Signatures of coherent population trapping of Mo spin states in $6 \mathrm{H}-\mathrm{SiC}$. Two-laser spectroscopy of the $L_{1}$ peak in the PLE signals reveals a dipped structure in the peak at several combinations of probe-beam and control-beam power. This results from coherent population trapping (CPT) upon $\Lambda$-scheme driving. Temperature, magnetic field orientation and magnitude, and laser powers, were as labeled. The data are offset vertically for clarity. Solid lines are fits of a theoretical model of CPT (see main text). The inset shows the normalized CPT feature depths

contribution from an effect linked to the spread in mass for Mo isotopes in natural abundance (nearly absent for the mentioned vanadium case), compatible with elongating spin coherence via spin-echo techniques. In addition, their work showed that the hyperfine coupling to the impurity nuclear spin can be resolved. There is thus clearly a prospect for storage times in quantum memory applications that are considerably longer than $T_{2}^{*}$.

\section{DISCUSSION}

The anisotropic behavior of the g-factor that we observed for Mo was also observed for vanadium and titanium in the EPR studies by Baur et al. ${ }^{33}$ (they observed $g_{\|} \approx 1.7$ and $g_{\perp}=0$ for the ground state). In these cases the transition metal has a single electron in its $3 \mathrm{~d}$ orbital and occupies the hexagonal $(h) \mathrm{Si}$ substitutional site. We show in Supplementary Information section 7 that the origin of this behavior can be traced back to a combination of a crystal field with $C_{3 v}$ symmetry and spin-orbit coupling for the specific case of an ion with one electron in its d-orbital.

The correspondence of this behavior with what we observe for the Mo impurity identifies that our materials have Mo impurities present as $\mathrm{Mo}^{5+}\left(4 d^{1}\right)$ systems residing on a hexagonal $h$ silicon substitutional site. In this case of a hexagonal $(h)$ substitutional site, the molybdenum is bonded in a tetrahedral geometry, sharing four electrons with its nearest neighbors. For $\mathrm{Mo}^{5+}\left(4 \mathrm{~d}^{1}\right)$ the defect is then in a singly ionized $+|e|$ charge state ( $e$ denotes the elementary charge), due to the transfer of one electron to the p-type SiC host material.

An alternative scenario for our type of Mo impurities was recently proposed by Ivády et al. $^{35}$. They proposed, based on theoretical work, ${ }^{35}$ the existence of the asymmetric split-vacancy (ASV) defect in SiC. An ASV defect in SiC occurs when an impurity occupies the interstitial site formed by adjacent silicon and carbon vacancies. The local symmetry of this defect is a distorted octahedron with a threefold symmetry axis in which the strong g-factor anisotropy $\left(g_{\perp}=0\right)$ may also be present for the $S=1 /$ 2 state. $^{50}$ Considering six shared electrons for this divacancy 
environment, the $\mathrm{Mo}^{5+}\left(4 \mathrm{~d}^{1}\right)$ Mo configuration occurs for the singly charged $-|e|$ state. For our observations this is a highly improbable scenario as compared to one based on the $+|e|$ state, given the p-type $\mathrm{SiC}$ host material used in our work. We thus conclude that this scenario by lvády et al. does not occur in our material. Interestingly, niobium defects have been shown to grow in this ASV configuration, ${ }^{52}$ indicating there indeed exist large varieties in the crystal symmetries involved with transition metal defects in SiC. This defect displays $S=1 / 2$ spin with several optical transitions between 892 and $897 \mathrm{~nm}$ in $4 \mathrm{H}-\mathrm{SiC}$ and between 907 and $911 \mathrm{~nm}$ in $6 \mathrm{H}-\mathrm{SiC}^{52}$

Another defect worth comparing to is the aforementioned chromium defect, studied by Koehl et al. ${ }^{37}$ Like Mo in $\mathrm{SiC}$, the $\mathrm{Cr}$ defect is located at a silicon substitutional site, thus yielding a $3 d^{2}$ configuration for this defect in its neutral charge state. The observed $S=1$ spin state has a zero-field splitting parameter of $6.7 \mathrm{GHz} .^{37}$ By employing optically detected magnetic resonance techniques they measured an inhomogeneous spin coherence time $T_{2}^{*}$ of $37 \mathrm{~ns}^{37}$ which is considerably shorter than observed for molybdenum in the present work. Regarding spin-qubit applications, the exceptionally low phonon-sideband emission of $\mathrm{Cr}$ seems favorable for optical interfacing. However, the optical lifetime for this $\mathrm{Cr}$ configuration $\left(146 \mu \mathrm{s}^{37}\right)$ is much longer than that of the Mo defect we studied, hampering its application as a bright emitter. It is clear that there is a wide variety in optical and spin properties throughout transition-metal impurities in $\mathrm{SiC}$, which makes up a useful library for engineering quantum technologies with spin-active color centers.

We have studied ensembles of molybdenum defect centers in $6 \mathrm{H}$ and $4 \mathrm{H}$ silicon carbide with $1.1521 \mathrm{eV}$ and $1.1057 \mathrm{eV}$ transition energies, respectively. The ground-state and excited-state spin of both defects was determined to be $S=1 / 2$ with large $g$-factor anisotropy. Since this is allowed in hexagonal symmetry, but forbidden in cubic, we find this to be consistent with theoretical descriptions that predict that Mo resides at a hexagonal lattice site in $4 \mathrm{H}-\mathrm{SiC}$ and $6 \mathrm{H}-\mathrm{SiC}^{35,38}$ and our p-type host environment strongly suggests that this occurs for Mo at a silicon substitutional site. We used the measured insight in the $S=1 / 2$ spin Hamiltonians for tuning control schemes where two-laser driving addresses transitions of a $\Lambda$ system, and observed CPT for such cases. This demonstrates that the Mo defect and similar transitionmetal impurities are promising for quantum information technology. In particular for the highly analogous vanadium color center, engineered to be in $\mathrm{SiC}$ material where it stays in its neutral $\mathrm{V}^{4+}$ $\left(3 d^{1}\right)$ charge state, this holds promise for combining $S=1 / 2$ spin coherence with operation directly at telecom wavelengths.

\section{METHODS}

\section{Materials}

The samples used in this study were $\sim 1 \mathrm{~mm}$ thick epilayers grown with chemical vapor deposition, and they were intentionally doped with Mo during growth. The PL signals showed that a relatively low concentration of tungsten was present due to unintentional doping from metal parts of the growth setup (three PL peaks near $1.00 \mathrm{eV}$, outside the range presented in Fig. 1a). The concentration of various types of (di)vacancies was too low to be observed in the PL spectrum that was recorded. For more details see ref. ${ }^{42}$

\section{Cryostat}

During all measurements, the sample was mounted in a helium flow cryostat with optical access through four windows and equipped with a superconducting magnet system.

\section{Photoluminescence (PL)}

The PL spectrum of the $6 \mathrm{H}-\mathrm{SiC}$ sample was measured by exciting the material with an $892.7 \mathrm{~nm}$ laser, and using a double monochromator equipped with infrared-sensitive photomultiplier. For the $4 \mathrm{H}-\mathrm{SiC}$ sample, we used a $514.5 \mathrm{~nm}$ excitation laser and an FTIR spectrometer.

\section{Photoluminescence Excitation (PLE)}

The PLE spectrum was measured by exciting the defects using a CW diode laser tunable from $1050 \mathrm{~nm}$ to $1158 \mathrm{~nm}$ with linewidth below $50 \mathrm{kHz}$, stabilized within $1 \mathrm{MHz}$ using feedback from a HighFinesse WS-7 wavelength meter. The polarization was linear along the sample $c$-axis. The laser spot diameter was $\sim 100 \mu \mathrm{m}$ at the sample. The PL exiting the sample sideways was collected with a high-NA lens, and detected by a single-photon counter. The peaks in the PLE data were typically recorded at a rate of about $10 \mathrm{kcounts} / \mathrm{s}$ by the single-photon counter. We present PLE count rates in arb.u. since the photon collection efficiency was not well defined, and it varied with changing the angle $\phi$. For part of the settings we placed neutral density filters before the single-photon counter to keep it from saturating. The excitation laser was filtered from the PLE signals using a set of three $1082 \mathrm{~nm}$ (for the $4 \mathrm{H}-\mathrm{SiC}$ case) or $1130 \mathrm{~nm}$ (for the $6 \mathrm{H}-$ $\mathrm{SiC}$ case) longpass interference filters. PLE was measured using an ID230 single-photon counter. Additionally, to counter charge state switching of the defects, a $770 \mathrm{~nm}$ re-pump beam from a tunable pulsed Ti:sapphire laser was focused at the same region in the sample. Laser powers as mentioned in the main text.

\section{Two-laser characterization}

The PLE setup described above was modified by focusing a detuned laser beam to the sample, in addition to the present beams. The detuned laser field was generated by splitting off part of the stabilized diode laser beam. This secondary beam was coupled into a single-mode fiber and passed through an electro-optic phase modulator in which an RF signal (up to $\sim 5 \mathrm{GHz}$ ) modulated the phase. Several sidebands were created next to the fundamental laser frequency, the spacing of these sidebands was determined by the RF frequency. Next, a Fabry-Pérot interferometer was used to select one of the first-order sidebands (and it was locked to the selected mode). The resulting beam was focused on the same region in the sample as the original PLE beams (diode laser and re-pump) with similar spot size and polarization along the sample $c$-axis. Laser powers were as mentioned in the main text. Small rotations of the $c$-axis with respect to the magnetic field were performed using a piezo-actuated goniometer with 7.2 degrees travel.

\section{Data processing}

For all graphs with PLE data a background count rate is subtracted from each line, determined by the minimum value of the PLE in that line (far away from resonance features). After this a fixed vertical offset is added for clarity. For each graph, the scaling is identical for all lines within that graph.

\section{DATA AVAILABILITY}

The data sets generated and analyzed during the current study are available from the corresponding author upon reasonable request.

\section{ACKNOWLEDGEMENTS}

We thank A. Gali for discussions and M. de Roosz, J. G. Holstein, T. J. Schouten, and H. Adema for technical support. Early discussions with Prof. Erik Janzén leading to initiation of this study are gratefully acknowledged. Financial support was provided by ERC Starting Grant 279931, the Zernike Institute BIS program, the Swedish Research Council grants VR 2016-04068 and VR 2016-05362, and the Carl-Trygger Stiftelse för Vetenskaplig Forskning grant CTS 15:339.

\section{AUTHOR CONTRIBUTIONS}

The project was initiated by C.H.v.d.W., O.V.Z., I.G.I., and N.T.S. SiC materials were grown and prepared by A.E. and B.M. Experiments were performed by T.B., G.J.J.L., and O.V.Z., except for the PL measurements which were done by A.G. and I.G.I. Data analysis was performed by T.B., G.J.J.L., C.G., O.V.Z., F.H., R.W.A.H., and C.H.W. T.B., G.J.J. L., and C.H.W. had the lead on writing the paper, and T.B. and G.J.J.L. are co-first author. All authors read and commented on the manuscript. 


\section{ADDITIONAL INFORMATION}

Supplementary information accompanies the paper on the npj Quantum Information website (https://doi.org/10.1038/s41534-018-0097-8).

Competing interests: The authors declare no competing interests.

Publisher's note: Springer Nature remains neutral with regard to jurisdictional claims in published maps and institutional affiliations.

\section{REFERENCES}

1. Gao, W., Imamoglu, A., Bernien, H. \& Hanson, R. Coherent manipulation, measurement and entanglement of individual solid-state spins using optical fields. Nat. Photonics 9, 363 (2015).

2. Togan, E. et al. Quantum entanglement between an optical photon and a solidstate spin qubit. Nature 466, 730 (2010).

3. Dolde, F. et al. High-fidelity spin entanglement using optimal control. Nat. Commun. 5, 3371 (2014).

4. Klimov, P. V., Falk, A. L., Christle, D. J., Dobrovitski, V. V. \& Awschalom, D. D. Quantum entanglement at ambient conditions in a macroscopic solid-state spin ensemble. Sci. Adv. 1, e1501015 (2015).

5. Hensen, B. et al. Loophole-free Bell inequality violation using electron spins separated by 1.3 kilometres. Nature 526, 682-686 (2015)

6. Kalb, N. et al. Entanglement distillation between solid-state quantum network nodes. Science 356, 928-932 (2017).

7. Budker, D. \& Romalis, M. Optical magnetometry. Nat. Phys. 3, 227-234 (2007).

8. Balasubramanian, G. et al. Nanoscale imaging magnetometry with diamond spins under ambient conditions. Nature 455, 648-651 (2008).

9. Dolde, F. et al. Electric-field sensing using single diamond spins. Nat. Phys. 7, 459-463 (2011).

10. Kraus, $\mathrm{H}$. et al. Magnetic field and temperature sensing with atomic-scale spin defects in silicon carbide. Sci. Rep. 4, 5303 (2014).

11. Bonato, C. et al. Optimized quantum sensing with a single electron spin using real-time adaptive measurements. Nat. Nanotechnol. 11, 247-252 (2016).

12. Dobrovitski, V., Fuchs, G., Falk, A., Santori, C. \& Awschalom, D. Quantum control over single spins in diamond. Annu. Rev. Condens. Matter Phys. 4, 23-50 (2013).

13. Childress, L. \& Hanson, R. Diamond NV centers for quantum computing and quantum networks. MRS Bull. 38, 134-138 (2013).

14. Doherty, M. W., Manson, N. B., Delaney, P. \& Hollenberg, L. C. The negatively charged nitrogen-vacancy centre in diamond: the electronic solution. New J. Phys. 13, 025019 (2011).

15. Maze, J. et al. Properties of nitrogen-vacancy centers in diamond: the group theoretic approach. New J. Phys. 13, 025025 (2011).

16. Thiering, G. \& Gali, A. Ab initio calculation of spin-orbit coupling for an NV center in diamond exhibiting dynamic Jahn-Teller effect. Phys. Rev. B 96, 081115 (2017).

17. Radnaev, A. et al. A quantum memory with telecom-wavelength conversion. Nat. Phys. 6, 894-899 (2010).

18. Zaske, S. et al. Visible-to-telecom quantum frequency conversion of light from a single quantum emitter. Phys. Rev. Lett. 109, 147404 (2012).

19. Dréau, A., Tcheborateva, A., El Mahdaoui, A., Bonato, C. \& Hanson, R. Quantum frequency conversion of single photons from a nitrogen-vacancy center in diamond to telecommunication wavelengths. Phys. Rev. Appl. 9, 064031 (2018).

20. Weber, J. et al. Quantum computing with defects. Proc. Natl Acad. Sci. USA 107, 8513 (2010).

21. Weber, J. et al. Defects in SiC for quantum computing. J. Appl. Phys. 109, 102417 (2011).

22. Von Bardeleben, $\mathrm{H}$. et al. NV centers in $3 \mathrm{C}, 4 \mathrm{H}$, and $6 \mathrm{H}$ silicon carbide: a variable platform for solid-state qubits and nanosensors. Phys. Rev. B 94, 121202 (2016).

23. Zargaleh, $S$. et al. Evidence for near-infrared photoluminescence of nitrogen vacancy centers in 4H-SiC. Phys. Rev. B 94, 060102 (2016).

24. Friedrichs, P., Kimoto, T., Ley, L. \& Pensl, G. Silicon Carbide 1 (Wiley, Weinheim, 2010).

25. Song, B.-S., Yamada, S., Asano, T. \& Noda, S. Demonstration of two-dimensional photonic crystals based on silicon carbide. Opt. Express 19, 11084-11089 (2011).

26. Koehl, W. F., Buckley, B. B., Heremans, F. J., Calusine, G. \& Awschalom, D. D. Room temperature coherent control of defect spin qubits in silicon carbide. Nature 479, 84-87 (2011).

27. Christle, D. J. et al. Isolated electron spins in silicon carbide with millisecond coherence times. Nat. Mater. 14, 160 (2015).
28. Zwier, O. V., O'Shea, D., Onur, A. R. \& van der Wal, C. H. All-optical coherent population trapping with defect spin ensembles in silicon carbide. Sci. Rep. $\mathbf{5}$ 10931 (2015).

29. Riedel, D. et al. Resonant addressing and manipulation of silicon vacancy qubits in silicon carbide. Phys. Rev. Lett. 109, 226402 (2012).

30. Kraus, $\mathrm{H}$. et al. Room-temperature quantum microwave emitters based on spin defects in silicon carbide. Nat. Phys. 10, 157 (2014).

31. Widmann, M. et al. Coherent control of single spins in silicon carbide at room temperature. Nat. Mater. 14, 164 (2015).

32. Hobgood, H. M. et al. Semi-insulating $6 \mathrm{H}-\mathrm{SiC}$ grown by physical vapor transport. Appl. Phys. Lett. 66, 1364 (1995).

33. Baur, J., Kunzer, M. \& Schneider, J. Transition metals in SiC polytypes, as studied by magnetic resonance techniques. Phys. Status Solidi A 162, 153-172 (1997).

34. Magnusson, B. \& Janzén, E. Optical characterization of deep level defects in SiC. Mater. Sci. Forum 483, 341-346 (2005).

35. Ivády, V., Gällström, A., Son, N. T., Janzén, E. \& Gali, A. Asymmetric split-vacancy defects in SiC polytypes: a combined theoretical and electron spin resonance study. Phys. Rev. Lett. 107, 195501 (2011).

36. Son, $\mathrm{N}$. T. et al. Electron paramagnetic resonance and theoretical studies of $\mathrm{Nb}$ in 4H-and 6H-SiC. J. Appl. Phys. 112, 083711 (2012).

37. Koehl, W. F. et al. Resonant optical spectroscopy and coherent control of $\mathrm{Cr} 4+$ spin ensembles in SiC and GaN. Phys. Rev. B 95, 035207 (2017).

38. Csóré, A., Gällström, A., Janzén, E. \& Gali, Á. Investigation of Mo defects in 4H-SiC by means of density functional theory. Mater. Sci. Forum 858, 261-264 (2016).

39. Dombrowski, K. et al. Identification of molybdenum in $6 \mathrm{H}-\mathrm{SiC}$ by magnetic resonance techniques. Phys. Rev. B 54, 7323 (1996).

40. Kimoto, T., Nakajima, T., Matsunami, H., Nakata, T. \& Inoue, M. Formation of semiinsulating $6 \mathrm{H}-\mathrm{SiC}$ layers by vanadium ion implantations. Appl. Phys. Lett. 69, 1113 (1996).

41. Gällström, A., Magnusson, B. \& Janzén, E. Optical identification of Mo related deep level defect in 4H and 6H SiC. Mater. Sci. Forum 615, 405-408 (2009).

42. Gällström, A. Optical characterization of deep level defects in SiC. PhD dissertation, No. 1674, ISSN 0345-7524 Linköping University (2015).

43. Manson, N. \& Wei, C. Transient hole burning in NV centre in diamond. J. Lumin 58, 158-160 (1994)

44. Santori, C. et al. Coherent population trapping in diamond NV centers at zero magnetic field. Opt. Express 14, 7986-7994 (2006).

45. Fleischhauer, M., Imamoglu, A. \& Marangos, J. P. Electromagnetically induced transparency: optics in coherent media. Rev. Mod. Phys. 77, 633 (2005).

46. Kimble, H. J. The quantum internet. Nature 453, 1023 (2008).

47. Zwier, O. V. Two-laser spectroscopy and coherent manipulation of color-center spin ensembles in silicon carbide. Zernike Institute PhD thesis series, ISSN 15701530, University of Groningen (2016).

48. Beha, K., Batalov, A., Manson, N. B., Bratschitsch, R. \& Leitenstorfer, A. Optimum photoluminescence excitation and recharging cycle of single nitrogen-vacancy centers in ultrapure diamond. Phys. Rev. Lett. 109, 097404 (2012).

49. Wolfowicz, G. et al. Optical charge state control of spin defects in $4 \mathrm{H}-\mathrm{SiC}$. Nat Commun. 8, 1876 (2017)

50. Abragam, A. \& Bleaney, B. Electron Paramagnetic Resonance Of Transition Ions. (Clarendon Press, Oxford, 1970). International series of monographs on physics.

51. Doherty, M. W. et al. The nitrogen-vacancy colour centre in diamond. Phys. Rep. 528, 1-45 (2013).

52. Gällström, A. et al. Optical properties and Zeeman spectroscopy of niobium in silicon carbide. Phys. Rev. B 92, 075207 (2015).

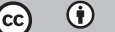

Access This article is licensed under a Creative Commons Attribution 4.0 International License, which permits use, sharing, adaptation, distribution and reproduction in any medium or format, as long as you give appropriate credit to the original author(s) and the source, provide a link to the Creative Commons license, and indicate if changes were made. The images or other third party material in this article are included in the article's Creative Commons license, unless indicated otherwise in a credit line to the material. If material is not included in the article's Creative Commons license and your intended use is not permitted by statutory regulation or exceeds the permitted use, you will need to obtain permission directly from the copyright holder. To view a copy of this license, visit http://creativecommons. org/licenses/by/4.0/.

(c) The Author(s) 2018 